\title{
„Da gibt es Sachen, die macht man einfach nicht““ - Deutungsmuster von Banken im Kontext von Nachhaltigkeit und Krise
}

\author{
Stefanie Hiß • Gesa Griese • Sebastian Nagel
}

Online publiziert: 17. September 2018

(C) Der/die Autor(en) 2018

Zusammenfassung Die Banken in Deutschland befinden sich in einer tiefgreifenden Legitimationskrise. Im Zuge von Finanz- und Wirtschaftskrisen, Bankenzusammenbrüchen und -Rettungspaketen sowie neuer Banken-Aufsicht und strengeren Regulierungsvorschriften haben die Banken erheblich an Reputation eingebüßt und an Vertrauen verloren. Der vorliegende Beitrag untersucht mithilfe einer Deutungsmusteranalyse, wie sich Banken unter Bezugnahme auf das gesellschaftliche Leitbild der Nachhaltigkeit in diesen Zeiten zu legitimieren versuchen. In Anknüpfung an das theoretische Konzept der gesellschaftlichen Legitimität in der neo-institutionalistischen Organisationstheorie sowie basierend auf diskursiven Interviews mit 18 Vertreterinnen und Vertretern deutscher Banken haben wir drei Deutungsmuster rekonstruiert: a) die Abgrenzung von „schlechten “ Banken, das die Abkehr von profitmaximierenden Geschäftsmodellen sowie die moralische Überlegenheit nachhaltigen Bankwesens beinhaltet, b) Verantwortung für die Gesellschaft, das die Rolle und Verantwortung von Banken als gesellschaftlich relevante Akteure und für gesellschaftlichen Wandel thematisiert, und c) ,Richtige“ Rendite, das anzeigt, dass Banken zur Legitimation nach dem angemessenen Verhältnis von Wertorientierung und Rendite suchen. Rendite ist zwar ökonomisch notwendig und legitimitätsstif\footnotetext{
gleichermaßen für beiderlei Geschlecht.

S. Hiß $(\bowtie) \cdot$ G. Griese $\cdot$ S. Nagel

Lehrstuhl Soziologie, Schwerpunkt Märkte, Organisationen, Governance,

Friedrich-Schiller-Universität Jena

Bachstr. 18k, 07743 Jena, Deutschland

E-Mail: stefanie.hiss@uni-jena.de

G. Griese

E-Mail: gesa.griese@uni-jena.de

S. Nagel

E-Mail: sebastian.nagel@uni-jena.de
}

Ein Hinweis vorab: Aus Gründen der besseren Lesbarkeit wird auf die gleichzeitige Verwendung männlicher und weiblicher Sprachformen verzichtet. Sämtliche Personenbezeichnungen gelten 
tend, darf aber nicht als Selbstzweck verstanden werden. Banken, die im Zuge der Krise diskreditiert wurden, können sich durch den Bezug zur Nachhaltigkeit so auf ein Bankwesen besinnen, das gesellschaftliche Legitimität zu versprechen scheint.

Schlüsselwörter Banken · Nachhaltigkeit · Deutungsmuster · Legitimität · Finanzkrise

\title{
"There are Things you Simply do not do"-Interpretative Patterns of Banks in the Context of Sustainability and Crisis
}

\begin{abstract}
Banks in Germany are experiencing a profound legitimacy crisis. In the wake of financial and economic crises, failures, and bailouts, as well as new oversight and stricter regulations, banks have lost the reputation and trust they once had. The present article uses an analysis of interpretative patterns to examine how banks seek to legitimize themselves in times of crisis, with reference to sustainability as a guiding principle of society. Following the concept of social legitimacy by new institutionalism in sociology, and based on discursive interviews with 18 representatives of German banks, we reconstructed three interpretative patterns: a) the differentiation from "bad" banks, which abandons profit-maximizing business models and promotes the moral superiority of sustainable banking, b) responsibility for society, which promotes the role and responsibility of banks as socially relevant actors that spur social change, and c) "right" return, whereby banks seek an appropriate relationship between their value orientation and a return on investments. Although a return on investment is economically necessary and confers legitimacy, it may not be understood as an end in itself. Banks discredited during the crisis can refocus on a banking model which promises social legitimacy by referring to sustainability.
\end{abstract}

Keywords Banks $\cdot$ Sustainability $\cdot$ Interpretative patterns $\cdot$ Legitimacy $\cdot$ Financial crisis

\section{Einleitung}

Als Folge der Finanzkrise, die 2007 mit dem Platzen der Immobilienblase in den USA begann, hatten zahlreiche Banken mit erheblichen Schwierigkeiten zu kämpfen. Während Lehman Brothers und Washington Mutual nur die prominentesten Banken sind, die im Herbst 2008 in den USA zusammenbrachen, waren auch etliche deutsche Kreditinstitute von den Auswirkungen der Krise betroffen. Die Finanzkrise, so scheint es, war nur der Auftakt für eine Reihe von Herausforderungen, mit denen Banken seit einigen Jahren konfrontiert sind. Es folgten Vertrauensverluste im Interbanken-Markt, die europäische Staatsschuldenkrise, neue regulatorische Rahmenbedingungen für Banken, die Niedrigzinspolitik der Notenbanken sowie die juristische Aufarbeitung der Finanzkrise, verbunden mit Strafzahlungen in den USA und in Europa. Banken stecken zweifelsohne in der Krise. Aufgrund ihrer maßgeblichen Beteiligung an der Entstehung der Finanzkrise sind Banken nicht nur wirtschaftlich bedroht, sondern auch erheblicher öffentlicher Kritik ausgesetzt. Mit Fragen danach, 
wozu es Banken überhaupt braucht oder für wen eine Bank eigentlich da sein sollte, steht auch die gesellschaftliche Legitimität von Banken zur Disposition (Rethel und Sinclair 2012). Als Unternehmen sind Banken zwar Teil von Marktprozessen, befinden sich als gesellschaftliche Akteure jedoch auch in Aushandlungsprozessen mit der Gesellschaft darüber, wie man als Bank agieren kann und sollte (Hiß und Nagel 2017; Wendt 2016).

In diesem Beitrag untersuchen wir mithilfe einer Deutungsmusteranalyse (Lüders und Meuser 1997; Oevermann 2001a), wie sich Banken als Reaktion auf diese Krisenprozesse zu legitimieren versuchen. Wir nehmen nicht die Veränderungen im Geschäftsablauf in den Blick, sondern rekonstruieren gegenwärtige Deutungsmuster, die die Handlungsorientierungen von Banken in krisenhaften Zeiten aufzeigen können. Basierend auf diskursiven Interviews mit Vertretern von 18 deutschen Banken rekonstruieren wir Deutungsmuster, die als Verbindungsglied von Struktur und Handlung zwischen dem objektiven Handlungsproblem der infrage gestellten Legitimität von Banken und dessen subjektiver Bewältigung vermitteln. Obgleich Legitimationsversuche auf zahlreichen Ebenen möglich sind (Suchman 1995), sei es durch die Schaffung neuer Strukturen oder Produkte, arbeiten wir hier mit den Deutungsmustern die kollektiven Sinngehalte heraus. Diese stellen trotz des funktionalen Bezugs zum objektiven Handlungsproblem, wie etwa der Delegitimierung und Dysfunktion von bestimmten Finanzprodukten, eine eigene Dimension sozialer Wirklichkeit dar (Meuser und Sackmann 1992a, S. 19).

Unter Rückgriff auf das Konzept der Legitimität in der neo-institutionalistischen Organisationstheorie (Deephouse und Suchman 2008) nehmen wir mit Banken Organisationen in den Blick, deren Legitimität im Zuge der vielfältigen Krisen infrage gestellt worden ist. Um die zum Überleben der Organisation notwendige Legitimität aus ihrer institutionellen Umwelt zugesprochen zu bekommen, müssen sie aktiv auf die Krise reagieren. Welche Deutungen einem möglichen Handeln der Banken zu Legitimationszwecken vorausgehen, ist Gegenstand unserer Analyse.

Nachhaltigkeit stellt den Kontext unserer Analyse dar. Die Berücksichtigung ökologischer und sozialer Belange hat sich in den vergangenen Jahrzehnten als eine Legitimationsstrategie im globalen Finanzmarkt sowie im deutschen Bankwesen ausgebreitet (Hiß 2011). Die im Nachhaltigkeitskonzept enthaltene Normativität verspricht eine Auseinandersetzung mit normativen Fragen selbst bei konventionellen Banken, wodurch Rechtfertigungen und somit auch latente Deutungsmuster für die Rekonstruktion eher offensichtlich werden als in anderen Kontexten des Bankwesens.

Als Ergebnis der Analyse haben wir drei Deutungsmuster rekonstruiert, die entsprechend der Fragestellung allesamt auf die Bewältigung der Legitimationskrise zielen. Die Deutungsmuster geben Antworten darauf, was genau als Problem für die Legitimationskrise identifiziert wird, welche Werthaltungen sich zeigen und welche Handlungsregeln daraus abgeleitet werden können. Während das erste, Abgrenzung von ,schlechten “ Banken, das profitmaximierende Geschäftsmodell von Großbanken problematisiert und die moralische Überlegenheit nachhaltigen Bankwesens als eine mögliche Lösung darstellt, werden im zweiten Deutungsmuster, Verantwortung für die Gesellschaft, der Vertrauensverlust und die Verantwortungslosigkeit als Probleme identifiziert. Hier besteht die Lösung in einer Handlungsregel, die be- 
inhaltet, wieder Verantwortung zu übernehmen und sich somit als gesellschaftlich relevante Akteure darzustellen. Dies gipfelt schließlich darin, einen eigenen Anspruch als Treiber für gesellschaftlichen Wandel zu formulieren. Das dritte Deutungsmuster ,Richtige“ Rendite zeigt an, dass Banken zur Legitimation nach einem ausgewogenen Verhältnis von Wertorientierung und Rendite suchen, da das Profitsteigerungsmotiv und auch die konventionelle Rendite sowohl durch die Krise als auch durch den nachhaltigen Finanzmarkt in Frage gestellt werden. Rendite ist zwar ökonomisch notwendig und legitimitätsstiftend, dürfe aber nicht als Selbstzweck verstanden werden.

Im Folgenden gehen wir zunächst auf die vielfältigen Krisen ein, mit denen Banken derzeit zu kämpfen haben (Abschn. 2). Anschließend erörtern wir mit dem Legitimitätskonzept der neo-institutionalistischen Organisationstheorie unsere theoretische Perspektive (Abschn. 3); mit einem Blick auf Nachhaltigkeit als Legitimationsstrategie im Finanzmarkt begründen wir anschließend die Fokussierung unserer Erhebung (Abschn. 4). Im darauffolgenden Abschnitt erläutern wir unser Verständnis von Deutungsmusteranalyse und legen unser methodisches Vorgehen offen (Abschn. 5). Danach stellen wir als Ergebnis unserer Analyse die drei von uns rekonstruierten Deutungsmuster systematisch vor (Abschn. 6). Wir schließen mit einer Diskussion der Ergebnisse (Abschn. 7) und einem Fazit (Abschn. 8).

\section{Banken in der Krise}

Das deutsche Bankwesen ist durch ein Drei-Säulen-Modell charakterisiert, bestehend aus den privatwirtschaftlichen Geschäftsbanken, den öffentlich-rechtlichen Banken und den Genossenschaftsbanken (siehe dazu Sachverständigenrat 2008, S. 84 f.). Die privatwirtschaftlichen Geschäftsbanken sind üblicher Weise als Aktienoder Personengesellschaften verfasst und damit den Interessen ihrer Aktionäre oder Eigentümer verpflichtet. Die öffentlich-rechtlichen Banken sind dem Gesetz nach dem Gemeinwohl verpflichtet und agieren etwa als Landesbanken als Hausbanken der Bundesländer, verfolgen als Förderbanken einen öffentlichen Entwicklungsauftrag oder dienen als Sparkassen der Region und dem Mittelstand als Kreditgeber. Der Genossenschaftssektor ist durch seine besondere Eigentümerstruktur genossenschaftlicher Mitgliederanteile den Miteigentümern verpflichtet. Der Zweck von Genossenschaften ist zudem darauf ausgerichtet, die sozialen, ökologischen oder kulturellen Interessen ihrer Mitglieder durch einen gemeinschaftlichen Geschäftsbetrieb zu fördern ( $11 \mathrm{GenG})$.

Diese Vielfalt der deutschen Bankenlandschaft hat die Kreditinstitute jedoch nicht vor den tiefgreifenden Folgen der zahlreichen, aufeinanderfolgenden Krisen bewahren können. Der Krisenreigen beginnt im Sommer 2007 mit dem Platzen der Immobilienkrise in den USA (Rona-Tas und Hiß 2010). Nur rund ein Jahr später, im September 2008, führt diese Entwicklung zur Beinahe-Kernschmelze des weltweiten Finanzsystems. Zur Abmilderung der Krisenerscheinungen werden weltweit Rettungspakete aufgelegt, sowohl zur konkreten Rettung von Banken vor Zahlungsunfähigkeit als auch zur Ankurbelung der Wirtschaft (Münnich 2016; Weber und Schmitz 2011; Woll 2014). Die Banken müssen in dieser Zeit weltweit hohe Ab- 
schreibungen vornehmen sowie die sogenannten toxischen Papiere, die sich in den Bilanzen der Banken befinden und fast vollständig an Wert verloren haben, in sogenannte Bad Banks auslagern. In den Jahren 2010 und 2011 entwickelt sich die globale Wirtschaftskrise zu einer vornehmlich europäischen Währungs- und Schuldenkrise weiter. Die Stabilität des Euro ist in Gefahr, weshalb Euro-Rettungsschirme und -Stabilitätspakte eingerichtet werden. Im Jahr 2012 leitet die Europäische Zentralbank eine Senkung des Leitzinses ein, der im Jahr 2016 den historischen Tiefstand von 0,0\% erreicht. Gleichzeitig soll der Aufbau einer europäischen Bankenaufsicht, die Ende 2014 ihre Arbeit aufnimmt, eine Wiederholung der Krise vermeiden.

Dieser kurze Rückblick auf die Geschehnisse seit 2007 macht nur allzu deutlich, wie sehr das Vertrauen in die Banken und ihre Legitimität erschüttert worden ist. Auch wenn sich inzwischen, im Oktober 2017, das Krisengeschehen wieder beruhigt hat, werden die Banken nach wie vor mit Problemen konfrontiert und, auch jenseits der Finanzkrise, von Skandalen erschüttert, die kaum dazu beitragen, das angeschlagene Vertrauen zurückzugewinnen (Honegger et al. 2010). Die Debatte über exorbitant hohe Gehälter und Bonuszahlungen von Bankern und das Thema Personalabbau von Banken bestimmen immer wieder die Schlagzeilen. Insgesamt ist und bleibt zudem die finanzielle Lage der Banken prekär. Durch die historisch niedrigen Leitzinsen bietet das Zinsgeschäft eher geringe Erträge, wodurch das bisherige Geschäftsmodell ins Wanken gerät. Insgesamt befinden sich die deutschen Banken nach wie vor in einer tiefgreifenden Krise: das Vertrauen in die Institution Bank ging seit 2007 mehr und mehr verloren. Der erlittene Reputationsschaden ist erheblich. Mit unserer Untersuchung zeigen wir auf, welche Deutungen von Banken hergestellt werden, um ihre gesellschaftliche Legitimität zurückzugewinnen.

\section{Gesellschaftliche Legitimität}

Die neo-institutionalistische Organisationstheorie rückt die Notwendigkeit von gesellschaftlicher Legitimität für das Überleben einer Organisation in den Vordergrund. Wir untersuchen, welche Antworten deutsche Kreditinstitute auf die gegenwärtige Legitimationskrise des Bankwesens finden. Organisationen passen sich institutionalisierten Erwartungsstrukturen an, da sie dadurch als legitim erachtet und mit notwendigen Ressourcen aus ihrer Umwelt versorgt werden (Senge 2011; Walgenbach und Meyer 2008). Würde eine Bank die Erwartungen der verschiedenen Akteure ihrer Umwelt nicht erfüllen, wäre sie in ihrer Existenz bedroht: Entweder würden zum Beispiel Kunden, Vertriebspartner oder Mitarbeiter die Geschäftsbeziehung beenden oder der Gesetzgeber würde der Bank die Geschäftsgrundlage entziehen.

Indem die neo-institutionalistische Organisationstheorie die Offenheit von Organisationen gegenüber ihrer Umwelt in den Vordergrund rückt, richtet sie den Blick darauf, organisationale Strukturen und Praktiken durch den institutionellen Kontext von Organisationen zu erklären (DiMaggio und Powell 1983; Meyer und Rowan 1977). Beispielsweise lässt sich mit Blick auf den institutionellen Kontext demonstrieren, weshalb Unternehmen trotz ihrer Profitorientierung mit verschiedenen Maßnahmen gesellschaftliche Verantwortung übernehmen (Hiß 2006). Institu- 
tionen bilden ein gesellschaftliches Regelsystem, das die Handlungen von Akteuren ermöglicht und zugleich begrenzt (Jepperson 1991; Senge 2006). Gesetzliche Regelungen zum Eigentum und zu Unternehmen sowie regulatorische Bestimmungen zum Bankwesen schaffen die Grundlage für das Handeln von Banken; zugleich setzen sie diesen aber auch Grenzen. Neben expliziten Regelungen in Form von Gesetzen, können Institutionen auch in Form von gesellschaftlich geteilten Normen und Werten oder auf einer kulturell-kognitiven Ebene wirksam werden (Scott 2008). Die sich aus diesem Kontext ergebenden Erwartungen, bei Banken zum Beispiel im Hinblick auf eine zu erzielende Rendite, bilden die Grundlage für organisationale Legitimität (Deephouse und Suchman 2008).

In aller Regel werden Organisationen als legitim erachtet, wenn sie die ihnen zukommende Funktion in der Gesellschaft erfüllen (Walgenbach und Meyer 2008, S. 65). Während Universitäten legitim erscheinen, wenn sie neues Wissen schaffen und wissenschaftlichen Nachwuchs ausbilden, nehmen Banken, zumindest in krisenfreien Zeiten, ihre gesellschaftliche Funktion dann wahr, wenn sie Kapital für Investitionen bereitstellen, Kredite vergeben und Finanzgeschäfte abwickeln. Legitimität können Organisationen nicht selbst herstellen, da Legitimität ein relationales Konzept ist. Einer Organisation kann Legitimität von anderen zugesprochen werden, wenn sie von diesen als legitim wahrgenommen wird (Suchman 1995, S. 594). Organisationen erhalten dementsprechend die zum Überleben notwendige Legitimität von ihrer institutionellen Umwelt zuerkannt, wenn sie die an sie gestellten institutionalisierten Erwartungen erfüllen. Indem eine Organisation versucht, sich zu legimitieren, reagiert sie auf die von ihr wahrgenommene Erwartungshaltung. Die rekonstruierten Deutungsmuster zeigen daher auch an, wie Banken die Erwartungen ihrer institutionellen Umwelt interpretieren und welche Handlungen als Folge und im Rahmen dieser Deutungen zur Legitimation beitragen würden.

Um die Deutungsmuster nachvollziehbar zu machen, haben wir, neben einem sorgfältigen methodischen Vorgehen, den Fokus unseres empirischen Materials jedoch von vornherein begrenzt. Das Konzept der Nachhaltigkeit, das sich in den vergangenen Jahrzehnten als eine Legitimationsstrategie im globalen Finanzmarkt und im deutschen Bankwesen ausgebreitet hat, bildete den Rahmen unserer Untersuchung.

\section{Nachhaltigkeit als Legitimationsstrategie im Finanzmarkt}

Der Grundsatz aus der Forstwirtschaft, nicht mehr Holz zu schlagen und damit nicht mehr Ressourcen zu verbrauchen, als nachwachsen können, bildet bis heute den Kern des Nachhaltigkeitsverständnisses (Sächsische Carlowitz-Gesellschaft 2013). Gemäß dem sogenannten Nachhaltigkeitsdreieck sollen ökologische, soziale und ökonomische Belange gleichberechtigt berücksichtigt werden, um so eine Generationengerechtigkeit zu sichern. In diesem Sinne formuliert die BrundtlandKommission die wohl am häufigsten zitierte Definition einer nachhaltigen Entwicklung, ,welche die Bedürfnisse der gegenwärtigen Generation befriedigt, ohne die Möglichkeiten künftiger Generationen zu gefährden, ihre eigenen Bedürfnisse zu befriedigen“ (Hauff 1987, S. 47). Seit den 2000er Jahren werden in Deutschland 
Forderungen an Unternehmen laut, das Nachhaltigkeitsdreieck in ihrer Wertschöpfungskette zu berücksichtigen und damit aktiv und freiwillig soziale Verantwortung zu übernehmen (Hiß 2006). Mittlerweile finden wir eine Vielzahl an Siegel- und Zertifikatsinitiativen, freiwilligen Verhaltenskodizes oder Konventionen zur unternehmerischen Verantwortung (siehe u. a. Toppinen et al. 2015).

Nachhaltigkeit ist auch auf dem Finanzmarkt angekommen. Das nachhaltige Investieren erhielt besonderen Aufschwung seit den 1980er Jahren in den USA, als Universitäten durch studentische Proteste dazu gedrängt wurden, ihre zu Zeiten der Apartheid in Südafrika investierten Vermögenswerte von dort abzuziehen (Soule 1997). In Deutschland ermöglichte die mit dem Wandel vom koordinierten zum Finanzmarkt-Kapitalismus einhergehende verstärkte Nutzung marktbasierter Finanzinstrumente seit den 1990er Jahren (Beyer und Wolf 2014; Lütz 2017; Windolf 2005) eine Popularisierung der Berücksichtigung von ökologischen und sozialen Anlagekriterien (Hiß 2011; Hiß et al. 2018).

Während inzwischen nahezu alle Banken nachhaltige Produkte im Angebot haben (Wendt 2016), wurde dies zuvor lediglich von darauf spezialisierten Banken angeboten (Herzog et al. 2015). Die 1974 gegründete GLS-Bank entwickelte sich aus der Waldorf-Bewegung, die aus der Not der fehlenden Finanzierung für eine Rudolf-Steiner-Schule das Modell für eine Leih- und Schenkgemeinschaft entwickelte. Die 1984 gegründete Ökobank (seit 2000 in die GLS-Bank eingegliedert) wollte, motiviert durch die Umwelt-, Friedens- und Anti-Atomkraftbewegung, eine Kreditvergabe für ökologische und soziale Projekte ermöglichen, die damals über den existierenden Bankensektor nicht realisierbar war (Hiß 2012, S. 91; Stiehl 1989). In Abgrenzung vor allem zur Ökobank gründete sich 1994 die bis heute bestehende Umweltbank $A G$ mit einer privatwirtschaftlichen Rechtsform, um der Gewinnorientierung mehr Raum zu geben (Herzog et al. 2015, S. 33). Im Jahr 2002 wurde schließlich die Ethikbank als Zweigniederlassung der Volksbank Eisenberg in Thüringen aus der Taufe gehoben. Seit 2009 hat sich zudem die niederländische Triodos Bank $A G$ als größte europäische Nachhaltigkeitsbank in Deutschland etabliert.

Neben diesen explizit nachhaltig agierenden Alternativbanken gibt es weitere Kreditinstitute, die sich dem nachhaltigen Bankwesen zuordnen lassen. Die Bank für Sozialwirtschaft finanziert überwiegend soziale Einrichtungen, die ebenfalls häufig als Genossenschaften verfassten Kirchenbanken nähern sich mit ihrer christlich geprägten Wertorientierung dem Konzept der Nachhaltigkeit und einige Landesbanken lassen sich von Nachhaltigkeits-Ratingagenturen bewerten und veröffentlichen ihre Nachhaltigkeitsleistung. Das Konzept der Nachhaltigkeit hält somit inzwischen auf verschiedenen Wegen Einzug in den Finanzmarkt und fast den gesamten Bankensektor und kann zu dessen Legitimierung beitragen. Inwieweit Anspruch und Wirklichkeit im Hinblick auf Nachhaltigkeit übereinstimmen, kann hier jedoch nicht überprüft werden. 


\section{Deutungsmusteranalyse: Methode, Daten und Vorgehen}

Basis unserer Analyse sind diskursive Interviews mit Vertretern von 18 deutschen Banken, die wir zwischen Juni und Oktober 2016 telefonisch sowie vor Ort durchgeführt haben. Wir haben Interviews mit Banken aus allen drei Säulen des deutschen Bankensystems geführt: Geschäftsbanken, Genossenschaftsbanken sowie Sparkassen und Landesbanken; darunter waren auch Kirchenbanken, explizite Nachhaltigkeitsbanken und Förderbanken. In unserem Sample sind sechs der zehn größten deutschen Banken vertreten. Die rekonstruierten Deutungsmuster vermitteln zwischen dem gesellschaftlich objektiven Handlungsproblem der infrage gestellten Legitimität von Banken und dessen subjektiver Bewältigung (Lüders und Meuser 1997, S. 59; Oevermann 2001a, S. 5).

Deutungsmuster sind kollektive Sinngehalte mit normativer Geltungskraft. Es sind „strukturierte Argumentationszusammenhänge“ (Oevermann 2001a, S. 5), die auf einer tiefenstrukturellen Ebene angesiedelt sind, die Interpretation und Wahrnehmung von Individuen organisieren und ihnen Antworten auf soziale Strukturprobleme geben (Meuser und Sackmann 1992a, S. 15f.; Oevermann 2001b). Sie sind latent und deswegen für die Akteure selbst nur bedingt reflexiv verfügbar. Sie sind daher weder zu verwechseln mit individuellen Einstellungen, Erwartungen oder der subjektiven systematischen Interpretation der Umwelt des Handelnden (Oevermann 2001a, S. 4, 28) noch mit habituell verfestigten Sinndeutungen (Lüders und Meuser 1997, S. 59). Zudem sind Deutungsmuster stets kontextabhängig und mit unterschiedlich weitreichender Geltungskraft ausgestattet (Lüders und Meuser 1997, S. 59; Meuser 2011; Meuser und Sackmann 1992a, S. 19). Beispielhaft wäre das Deutungsmuster der Mutterliebe zu nennen, das sich im 18. Jahrhundert etablierte und bis heute das Dasein von Frauen und die Beziehung zum Kind bestimmt, indem es Deutungen und Normen über die Natur der Frau, ihre Persönlichkeitsmerkmale und die Mutter-Kind-Beziehung enthält (Schütze 1992).

Aufgrund konzeptioneller und methodologischer Unschärfe sowie einer fehlenden Systematik hinsichtlich der Methodik stellt die Deutungsmusteranalyse selbst eher ein forschungspragmatisch-heuristisches Konzept dar (Lüders 1991, S. 379 f.; siehe dazu auch entsprechende Fallstudien: Bögelein 2016; Markova 2013; Meuser und Sackmann 1992b; Opielka et al. 2010; Sachweh 2010). Wir rekonstruieren auf Basis der geführten Interviews Deutungsmuster, die aufzeigen, wie Banken mit ihrer infrage gestellten Legitimität umgehen und wie sie im Kontext Nachhaltigkeit versuchen, die Legitimationskrise zu bewältigen. Deutungsmuster mit den beinhaltenden Deutungen und Handlungsregeln erklären eben nicht nur Handeln, sondern legitimieren dieses auch und geben Aufschluss über ,inkorporiertes, sozial verankertes Rezeptwissen“ (Markova 2013, S. 99).

Wir haben für die Erhebung des Materials diskursive Interviews gewählt, da diese durch die unter anderem verstärkten Begründungsaufforderungen die latenten Deutungsmuster besser zutage treten lassen als beispielsweise Experten-Interviews (Ullrich 1999; Markova 2013, S. 121) oder öffentlich zugängliche Kommunikation. Diese Erhebungsmethode und die anschließende inhaltsanalytische thematische Codierung und Feincodierung sowie der synoptische Vergleich können eine ,tiefgehende hermeneutische Analyse“ ersetzen (Markova 2013, S. 121). Aufgrund der im 
Vorfeld vermuteten Bedeutung von Nachhaltigkeit als Legitimationsstrategie im Finanzmarkt haben wir die jeweils 60- bis 90-minütigen Interviews mit Vertretern von konventionellen und von explizit nachhaltigen Banken geführt; je nach Verortung des Themas waren das Vertreter von Investor Relations-, CSR- oder Nachhaltigkeitsabteilungen oder auch Vorstände oder Regionalleiter. ${ }^{1}$

Um die latenten Deutungen der Interviewten hervorzubringen, haben wir in den diskursiven Interviews verschiedene Befragungstechniken angewendet (siehe dazu Ullrich 1999, S. 437-439): Neben provokanten und hypothetischen Fragen forderten wir die Interviewten auch explizit zu Begründungen auf. Zudem haben wir anhand eines eigens entwickelten Diagramms die Interviewpartner mit der Aufgabe konfrontiert, verschiedene Banken, einschließlich ihrer eigenen, darauf zu verorten. Die Achsen des Diagramms umfassten das primäre Leitbild (renditeorientiert - werteorientiert) sowie die Nachhaltigkeitsorientierung (niedrig - hoch). Die Fragen zielten sowohl auf das Verständnis und den Impuls von Nachhaltigkeit ab als auch auf die Darstellung von Unterschieden zwischen konventionellem und nachhaltigem Bankwesen. Zudem wurden strukturelle Veränderungen abgefragt, Produkte thematisiert und Fragen zum Finanzmarkt sowie dessen Stabilität und Krisen mit eingebunden. Mithilfe der Grafik wurde auch die Konkurrenz betrachtet und Einschätzungen über das gesamte Bankwesen erbeten.

Die Untersuchung der transkribierten Interviews begann unter Verwendung der Analysesoftware MaxQDA 12 mit einer ersten thematischen Grobcodierung des gesamten Materials anhand der im verwendeten Interview-Leitfaden vorgegebenen Inhalte. Anschließend folgte eine inhaltliche Feincodierung von sämtlichen Aussagen ausgewählter Codes. Die sich in der Feincodierung ergebenden inhaltlichen Ausprägungen wurden danach anschließend in Gruppenarbeit inhaltlich geclustert. In einem weiteren Schritt wurden die so zusammengefassten Aussagen eines thematischen Codes entsprechend der Clusterung geordnet und nach Zuordnung der Interviews je nach Banktyp zu einem Set (Geschäfts-, Genossenschafts-, Kirchenund Landesbanken, Sparkassen und explizite Nachhaltigkeitsbanken) miteinander verglichen. Im letzten Schritt wurden auftauchende Verdichtungen und Hinweise auf Deutungsmuster in mehreren gemeinsamen Gruppenauswertungen rekonstruiert, unter anderem, indem wir weitere Zusammenfassungen über die thematischen Codes hinweg erstellt haben. Durch die vorangegangene intensive Codierung des Materials konnten immer wieder Interviewstellen hinzugezogen werden, um ein Deutungsmuster zu bestätigen, zu verfeinern oder zu korrigieren. Die Deutungsmuster sind aus dem Antwortverhalten zu unterschiedlichen Fragen rekonstruiert worden. Sie beinhalten somit nicht die Handlungspraxis an sich, sondern das dahinterliegende sozial inkorporierte Rezeptwissen. Interessant ist hier, dass trotz unterschiedlicher Rechtsformen der Banken und unterschiedlicher Ausformung der Handlungspraxis gemeinsame Bezugsrahmen gefunden werden konnten.

\footnotetext{
1 Wir haben auch Banken angefragt, die nicht offensiv mit Nachhaltigkeit werben, haben von diesen jedoch keine Interviewzusagen erhalten.
} 


\section{Empirische Ergebnisse}

Auf Basis der diskursiven Interviews haben wir im Rahmen der Deutungsmusteranalyse drei verschiedene Deutungsmuster aus dem gesamten Material rekonstruiert, die wir im Folgenden systematisch darstellen: a) Abgrenzung von ,schlechten “ Banken, b) Verantwortung für die Gesellschaft und c) „Richtige“ Rendite. Diese Deutungsmuster verweisen auf aktuelle Legitimationsstrategien und erklären Handeln durch die Offenlegung der dahinterliegenden Deutungsstruktur. Die Benennung der Deutungsmuster kann bereits zu verschiedenen Assoziationen, soll aber nicht zu vorschnellen Urteilen führen: Wichtig für das Verständnis ist die konkrete Operationalisierung. Anhand der ausführlichen Darstellung der einzelnen Bestandteile jedes Deutungsmusters wird die konkrete Bedeutung, unabhängig von der bloßen Benennung, nachvollziehbar. Zudem reproduzieren die Deutungsmuster keine Ansichten von einzelnen Banken oder von Säulen des Bankensystems, sondern verweisen auf die vorrangig zu findenden abstrakten überindividuellen Orientierungsmuster aller interviewten Banken.

Entsprechend unserer Frage danach, wie sich Banken als Reaktion auf die Krise legitimieren, zielen die rekonstruierten Deutungsmuster jeweils darauf ab, die Legitimationskrise der Banken zu bewältigen (siehe die Übersicht der Ergebnisse in Tab. 1). Wir zeigen deshalb die dem jeweiligen Deutungsmuster zugrunde liegende Problemdefinition als Ursache der Delegimitation auf, erläutern die damit verbundenen Wertvorstellungen und beschreiben die Handlungsregel, die sich für die Banken aus diesem Deutungsmuster ergibt.

\subsection{Abgrenzung von „schlechten“ Banken}

Insbesondere international agierende Großbanken mitsamt ihren Einstellungen und Praktiken dienen im ersten Deutungsmuster als negative Referenzfolie, um sich von diesen ,schlechten“ Banken abzugrenzen. Eine Abkehr von Profitmaximierung und den dazugehörigen als fragwürdig angesehenen Geschäftspraktiken sowie der Intransparenz wird als moralisch überlegen bewertet. Nachhaltigkeit bietet als normatives Konzept dazu eine Alternative und ermöglicht diese Abgrenzung. Als Reaktion auf diese Deutung ergibt sich für Banken folgende Handlungsregel: Grenze dich glaubwürdig von ,schlechten“ Banken $a b$. Im Folgenden werden die Problemdefinition, die Wertvorstellung und die Handlungsregel dieses Deutungsmusters anhand von ausgewählten Beispielen aufgezeigt.

\subsubsection{Problemdefinition}

Durch die Analyse des Interviewmaterials wird ein Problem offensichtlich, das für dieses Deutungsmuster bestimmend ist: Ein Geschäftsmodell von Banken, das auf Profitmaximierung setzt, wird mit Blick auf die Zukunft der Gesellschaft für problematisch gehalten; schließlich hat es auch in die Krise geführt. Indem diesem Ziel auf Seiten ,schlechter“ Banken vieles untergeordnet wird, werden falsche Anreize gesetzt, sodass kein Mehrwert für die Gesellschaft generiert werden kann. Die Begründungen und Erzählstrategien in verschiedenen Interviews drehen sich um 
Tab. 1 Übersicht der Ergebnisse der Deutungsmusteranalyse (eigene Darstellung)

\begin{tabular}{|c|c|c|c|}
\hline \multirow[t]{2}{*}{ Merkmal } & \multicolumn{3}{|l|}{ Deutungsmuster } \\
\hline & $\begin{array}{l}\text { Abgrenzung von } \\
\text { „schlechten“ Banken }\end{array}$ & $\begin{array}{l}\text { Verantwortung für die } \\
\text { Gesellschaft }\end{array}$ & „Richtige“ Rendite \\
\hline Problemdefinition & $\begin{array}{l}\text { Profitmaximierende } \\
\text { Geschäftsmodelle von } \\
\text { Großbanken haben kei- } \\
\text { nen gesellschaftlichen } \\
\text { Mehrwert }\end{array}$ & $\begin{array}{l}\text { Die Strukturen und } \\
\text { Denkweisen gegenwär- } \\
\text { tigen Wirtschaftens sind } \\
\text { risikobehaftet und ste- } \\
\text { hen einer nachhaltigen } \\
\text { Gesellschaft entgegen }\end{array}$ & $\begin{array}{l}\text { Banken müssen das } \\
\text { richtige Verhältnis } \\
\text { zwischen den ge- } \\
\text { gensätzlichen Polen } \\
\text { Renditestreben und } \\
\text { Wertorientierung finden }\end{array}$ \\
\hline Wertvorstellung & $\begin{array}{l}\text { Geschäftsmodelle der } \\
\text { Großbanken sind schäd- } \\
\text { lich; sie handeln un- } \\
\text { ethisch und riskieren } \\
\text { die Zukunft der Gesell- } \\
\text { schaft }\end{array}$ & $\begin{array}{l}\text { Banken sind gesell- } \\
\text { schaftlich relevante } \\
\text { Akteure, die Verant- } \\
\text { wortung übernehmen } \\
\text { und die Zukunft der Ge- } \\
\text { sellschaft mitgestalten } \\
\text { sollen }\end{array}$ & $\begin{array}{l}\text { Rendite ist legitim, } \\
\text { notwendig und legiti- } \\
\text { mitätsstiftend, aber darf } \\
\text { nicht als Selbstzweck } \\
\text { verfolgt werden }\end{array}$ \\
\hline Handlungsregel & $\begin{array}{l}\text { Grenze dich glaubwür- } \\
\text { dig von ,schlechten“ } \\
\text { Banken ab }\end{array}$ & $\begin{array}{l}\text { Nutze deinen Gestal- } \\
\text { tungsspielraum und } \\
\text { nimm Verantwortung } \\
\text { für die Gesellschaft } \\
\text { wahr }\end{array}$ & $\begin{array}{l}\text { Löse den Widerspruch } \\
\text { zwischen Rendite- und } \\
\text { Wertorientierung auf }\end{array}$ \\
\hline
\end{tabular}

eine implizite und durch das diskursive Interview auch explizite Abgrenzung zu Großbanken, die als negative Referenzfolie dienen.

Ackermann $^{2}$ hat mal gesagt, der Banker, der nicht $18 \%$ Nettorendite erwirtschaftet, ist kein guter Banker. Und jeder hat gewusst: „Das ist so ein Idiot!“ Weil, das gibt es nicht. Also, solche Geschäfte sind ... sowas wächst ... nur Krebs wächst so schnell, und nichts anderes. Und Krebs bringt uns um, und das bringt uns auch um. [...] [D]ie Grundausrichtung ... also, bei denen ist es so: Der Zweck heiligt jedes Mittel. [...] Also, die haben, alles was [...] und das ist bei uns halt, da sind wir vielleicht ein Stück weit auch eine kleinere [...] Bank, da gibt es ein paar Sachen, die macht man einfach nicht. (N 96-98) ${ }^{3}$

Nachhaltiges Bankwesen dient hier dazu, sich von diesen „schlechten“ Banken glaubhaft abzugrenzen, indem auf bestimmte Produkte, andere Geschäftsmodelle und vor allem andere Wertestandards verwiesen wird (auch E 21):

Ich glaube, das ist das größte Problem, dass diese Wertebasis, Wertvorstellung bei konventionellen Banken nicht vorhanden ist. (O 29)

Konventionelle Banken [...] haben eben immer diesen monetären Ansatz, wie die Gewinnmaximierung stattfinden kann. Und bei diesen Alternativbanken [...] stehen halt auch andere Ziele noch im Vordergrund. (G 21)

\footnotetext{
2 Josef Ackermann war zwischen 2002 und 2012 zunächst Vorstandssprecher und ab 2006 Vorstandsvorsitzender der Deutschen Bank AG.

${ }^{3}$ Die Zitatstellen werden anonymisiert dargestellt; jedem Interview wurde ein Buchstabe zugeordnet, dem jeweils die entsprechende Absatznummer des Zitats hinzugefügt wurde.
} 
Von daher denke ich mal, dass man nicht sagen kann, die sind per se alle unnachhaltig, aber ... ja, ich denke auch immer, solange viele noch in sehr fragwürdigen Bereichen sich engagieren, und es denen dann eigentlich egal ist, was dann mit betroffenen Menschen passiert, dann ist es auch nicht nachhaltig.

Wegen einer grundlegenden Ausrichtung auf Profitmaximierung und fehlenden Werten, werden entsprechende Banken nicht nur als problematisch erachtet, sondern deren Praktiken mit einer tödlichen Krankheit verglichen. Den Banken wird abgesprochen, eine Wertebasis jenseits von Rendite zu haben. Da deren Geschäftsmodelle als ein Auslöser der Krise identifiziert werden, wird ein „weiter so“ nicht als Lösung verstanden (O 29-33). Bei der Frage, wer nun zu den werteorientierten (,guten“) Banken gehört, zeigt sich, dass vor allem wenige Großbanken zu den „schlechten“ gezählt werden. Darauf aufbauend geht es in vielen Darstellungen darum zu zeigen, dass man selbst anders und nicht wie diese Banken sei. Zur Abgrenzung kann dann sowohl die eigene Nachhaltigkeitsausrichtung dienen als auch die Betonung der grundlegenden Andersartigkeit aufgrund des anderen Geschäftsmodells. Es geht insgesamt darum, glaubwürdige Beweise dafür aufzuzeigen (,[Nachhaltigkeit] wird gelebt“" O 29), dass man nicht zur falschen Seite gehört - und das, obwohl natürlich alle Banken weiterhin Renditeziele verfolgen (siehe Deutungsmuster 3).

\subsubsection{Wertvorstellung}

Die mit der Problemdefinition des Deutungsmusters verbundene Wertvorstellung besteht darin, dass "schlechte“ Banken ethisch nicht korrekt handeln, keine Wertehaltung haben und dadurch der Gesellschaft erheblichen Schaden zufügen. Diese Banken, so die Zuschreibung, nehmen unter anderem durch spekulative Geschäfte ihre gesellschaftliche Funktion als Bank nicht oder nicht angemessen wahr.

Die Wertvorstellung von den „schlechten“ Banken lässt sich daran verdeutlichen, welche fragwürdigen Geschäftspraktiken und intransparenten Prozessen ihnen zugeschrieben werden. So werden bestimmte Finanzgeschäfte dieser Banken abgelehnt, wie der Devisenhandel und der renditeorientierte Hochfrequenzhandel, weil sie auf moralischer Basis problematisch sind und an der Entstehung der Finanzkrise beteiligt waren (u. a. R 17). Die Kreditvergabe wird als moralisch überlegenes Bankgeschäft gesehen. Deshalb wird, trotz ähnlicher Rendite, das Geschäft dieser Banken im Vergleich zu nachhaltigen Banken als wenig wünschenswert dargestellt:

Während nachhaltige Banken im internationalen Durchschnitt über 70\% der Einlagen ihrer Kunden, also der Bilanzsumme, wieder in Krediten ausgeben und zwar an die Realwirtschaft, sind das bei systemrelevanten Banken unter $40 \%$. Was machen die mit dem Rest des Geldes? Und da liegt der Hase im Pfeffer. Das sind dann Spekulativgeschäfte, Eigenhandel, um die Rendite nach oben zu treiben. Das heißt, da passieren genau eigentlich die Dinge, die eigentlich relativ viele Leute, wenn sie mal drüber nachdenken, gar nicht so gut finden. Hochfrequenzhandel, Spekulation auf Lebensmittel oder sonstige Rohstoffe, die essenziell sind. (L 19) 
Ebenso werden intransparente Prozesse den „schlechten“ Banken zugeschrieben; es wird in erster Linie auf ,tiefgreifende Unterschiede“ hingewiesen, die zwischen den ,schlechten“ und den ,guten“ Banken bestehen. Insbesondere die Offenlegung von Investitionskriterien, die Transparenz über Geldflüsse in der Bank und die Vermeidung undurchsichtiger Spekulationsgeschäfte werden für die Beschreibung ,guter" Banken verwendet:

Also, wenn Sie jetzt auf diese nachhaltige Branche anspielen ... dann denke ich schon, dass dort die Spekulationsbereitschaft deutlich geringer ist. Weil man eben sagt, man will nicht mehr dieses hochriskante Geschäft haben, dieses undurchsichtige Geschäft. Ich denke, da geht es auch sehr viel um Transparenz, und zu zeigen, das machen wir mit eurem Geld. Also, Banken sind ja per se nicht dafür da zu spekulieren, sondern sind ja dafür da, Geld von Leuten, die es haben, an Leute zu geben, die es nicht haben. [...] Das ist das, warum diese Banken dann doch eher gegen so großspurige Spekulationen sind. (I 31-33) Ich glaube, [...] weil wir auch den Filter sehr deutlich publiziert haben, sehr transparent sind und versuchen, auch die Glaubwürdigkeit unseres Hauses dadurch zu erhöhen. [...] So, und das ist, glaube ich, ein Unterschied zu anderen, die einfach sagen: Wir haben Produktinseln und sagen, wir haben da unsere Nachhaltigkeitsfamilie, aber wir haben eben auch weiterhin das konventionelle Geschäft. Und wenn die veröffentlichen würden und die Zahl, wie viel machen sie konventionell und wie viel machen sie nachhaltig, dann sieht die Zahl schon anders aus. Wir können halt eben sagen bis zu einem sehr hohen Prozentsatz, das geht fast an die $100 \%$ ran, versuchen wir unsere Kriterien zu erfüllen. [...] Und das ist, glaube ich, schon ein Unterschied zu den anderen Banken, die solch eine Statistik nicht offen legen. (C 29)

\subsubsection{Handlungsregel}

Als mögliche Reaktion auf diese Deutung wird durch die Analyse eine Handlungsregel für Banken offensichtlich: Grenze dich glaubwürdig von ,schlechten “ Banken $a b$. Eine solche Abgrenzung bietet auf Basis der Wertvorstellung eine Problemlösung an, mit der Banken ihre Legitimationskrise überwinden können. Diese Handlungsregel zeigt sich vor allem an der Bedeutung, die Banken ihrer grundsätzlichen Andersartigkeit zusprechen. Ihr Fokus liegt auf der Konstruktion einer scharfen Grenze zwischen den ,guten“ und den „schlechten“ Banken. Indem sie sich selbst den ,guten“ Banken zurechnen, nehmen sie sich und ihr Handeln als legitim wahr. Nachhaltigkeit bietet scheinbar einen eindeutigen Beleg für diese Andersartigkeit und Wertehaltung.

Die interviewten Banken stellen sich nämlich als grundsätzlich nachhaltig dar; sie hätten immer schon mehr als nur Rendite im Sinn. Nachhaltigkeit oder Andersartigkeit wird wiederkehrend als ein Teil der DNA der Bank verstanden (u.a. E 31-34; I 37-38; Q 28, O 9, 15, 21; L 23), sodass eine Abgrenzung zu „,schlechten“ Banken dadurch selbst dann gelingt, wenn sich die Verfolgung von Nachhaltigkeit noch nicht in allen Geschäftspraktiken zeigt: 
[...] [B]ei uns ist ganz klar das Soziale im Vordergrund. [...] Das ist bei uns zu $100 \%$ drin in uns. Das heißt, das gab es hier nie anders. [...] Deswegen ist es momentan bei uns institutionalisiert nicht so, dass man sagen kann, Nachhaltigkeit, so wie man es in dem modernen Weg versteht, beeinflusst die Entwicklung unserer Produkte. Ich würde es aber wieder auch ein bisschen biologisch-genetisch erklären, es ist trotzdem ein großes Ziel von uns, weil wir uns schon immer genau in der Szene bewegen, die Nachhaltigkeit für mich eigentlich auch beschreibt. (R 9)

Also, wir sind eine rein ethisch-nachhaltige Bank. Wir machen nichts anderes. Also, für uns ist das nicht ein Zusatzaufgabengebiet, weil es jetzt erst als Modethema irgendwie dazu kam [...]. Uns ist die Verpflichtung auf die ethischnachhaltige Geldanlage sozusagen schon mit in die Wiege gelegt worden seit Gründung. (G 12)

Um ihre Andersartigkeit darzustellen, verweisen die Banken auf eine enge Verknüpfung ihres Geschäfts- und Eigentümermodells mit dem Nachhaltigkeitsgedanken. Während Förderbanken zur Darstellung ihrer Wesensverschiedenheit ihren gesetzlichen Auftrag betonen (Q 10) und Genossenschaftsbanken auf die Grundidee von Genossenschaften rekurrieren (D 29), rücken Sparkassen ihre Regionalität und eher geringe Renditeorientierung in den Vordergrund. Wiederum wird eine Großbank als negative Referenz verwendet:

Und womit kann man sich unterscheiden? Das ist unser regionales Engagement, das ist unser gesellschaftliches Engagement, das ist im Grunde unser nachhaltiges Engagement für die Region, angefangen von Arbeitsplätzen bis hin zu sozialen Themen und Umweltthemen. Und ich glaube, dass das eine Säule ist oder sein muss in Zukunft, um Menschen davon zu überzeugen, noch zu uns zu kommen. [...] Aus meiner Sicht hat die Sparkasse alle Chancen, weil, wo kommt sie her? [...] Wir sind ja nicht so renditeorientiert. Und diese Renditeziele der Deutschen Bank „Wir wollen mal 25\% Eigenkapitalrendite“, so was hatten wir nie. (K 93-95)

Gerade am letzten Beispiel kann man erkennen, dass sich, obwohl die Nachhaltigkeitsbemühungen der untersuchten Banken sehr unterschiedlich ausfallen, vor allem Einigkeit in deren Deutung über ihre moralische Überlegenheit gegenüber privaten Großbanken herrscht. Nachhaltigkeit eröffnet eine Sichtweise auf eigene moralische Stärken, die man gegenüber den Großbanken in Stellung bringen kann.

\subsection{Verantwortung für die Gesellschaft}

Im zweiten Deutungsmuster verstehen sich Banken als wichtige Akteure, denen Verantwortung für die Gesellschaft und für einen gesellschaftlichen Wandel zukommt. Einige Banken sehen sich in dieser Deutung als Gestalter, die bestehende Strukturen und Wirtschaftsweisen überwinden und die Gesellschaft positiv gestalten möchten. Trotz konträrer Ansichten hinsichtlich der legitimen Wertvorstellungen im Bankwesen offenbart sich darin die Reflexion über die eigene Verantwortlichkeit. Während im ersten Deutungsmuster das Feld der Banken in „gute“ und „schlechte“ 
Banken aufgeteilt wurde, geht es hier also um die Funktion und Rolle von Banken in der Gesellschaft. Die Handlungsregel lautet: Nutze deinen Gestaltungsspielraum und nimm Verantwortung für die Gesellschaft wahr oder zeige, wo und wie du Verantwortlichkeit lebst. Im Folgenden werden wiederum die Problemdefinition, die Wertvorstellung und die Handlungsregel dieses Deutungsmusters anhand von ausgewählten Beispielen erörtert.

\subsubsection{Problemdefinition}

Das dem Deutungsmuster zugrunde liegende Problem wird in bestehenden Strukturen und Denkweisen gegenwärtigen Wirtschaftens gesehen. Sie sind risikobehaftet, haben die jüngste Finanzkrise mitverursacht und stehen zudem einer nachhaltigen Gesellschaft entgegen. Die Problemdefinition des Deutungsmusters wird unter anderem bei der Diskussion um den Ausschluss bestimmter Investitionsfelder deutlich (u.a. O 35) oder bei Verweisen auf korrupte Praktiken und das Investmentbanking (u.a. R 17). Dass die vorherrschenden Denkweisen der Branche als problematisch wahrgenommen werden, lässt sich am Beispiel der Ziele aufzeigen, die Banken ausweisen. Dazu gehören die Rückkehr zum ursprünglichen Bankwesen, das Schaffen von Bewusstsein und die Aufklärung von Kunden und Gesellschaft über die Wirkung von Geld und die Notwendigkeit, das Denken zu verändern:

Und der Grundgedanke ist tatsächlich der, Banking in der ursprünglichsten Form durchzuführen. [...] investieren das in die Realwirtschaft, in Kredite, [...] da schränken wir uns eben noch ein auf Branchen, Sektoren oder Projekte, von denen wir sagen: „Die unterstützen den Wandel in der Gesellschaft und bieten positiven Wandel." (L 9)

F: Haben Sie das Gefühl, dass Sie [...] Politik machen? A: In gewissem Maße schon, weil wir ja schon damit sorgen ... oder mit unserem Angebot oder mit unserem Auftritt dafür sorgen, das Thema Nachhaltigkeit und Umweltschutz eigentlich in den Köpfen der Menschen zu verankern. [...] Und da leisten wir ... na gut, man kann es jetzt Aufklärungsarbeit nennen, aber da leisten wir, denke ich, schon einen guten Beitrag, genau wie die anderen Nachhaltigkeitsbanken. [...] Das gemeinsame Ziel ist einfach, die Gesellschaft nachhaltig umzustellen, sowohl Soziales als auch ökologisch. (I 216-223)

Kunden sollen dazu aktiviert werden, zu überdenken, was sie mit ihrem Geld eigentlich machen (M 4-9). Das Ziel sei es, Bewusstsein zu schaffen und dadurch auf Kunden einzuwirken ( $\mathrm{G}$ 22-24, 123):

Nee, eigentlich muss jeder, egal, ob er einen Cent hat oder ein paar Millionen, sich darüber Gedanken machen, was tue ich mit dem Geld. [...] Also Bewusstsein, Aufklärung, Aufwecken. (M 72-74)

\subsubsection{Wertvorstellung}

Die Wertvorstellung besteht darin, dass das Wahrnehmen gesellschaftlicher Verantwortung durch Banken moralisch geboten sei. Darüber hinaus wird eine nachhaltige Gesellschaft als erstrebenswert erachtet, auch weil „Nachhaltigkeit zunehmend 
Zukunftsfähigkeit meint" (A 43). Wie man sich jedoch verantwortlich zeigt, was die richtige Werthaltung ist, um verantwortungsvoll $\mathrm{zu}$ handeln, ist umstritten. Es herrscht die Ansicht, die eigenen Wertvorstellungen könnten nicht zum Maßstab für die Gesellschaft gemacht werden (u. a. B 49-53; R 44-47), weil es in einer pluralistischen Gesellschaft kaum möglich sei, allen Werten gerecht zu werden (u.a. A 81; B 59; D 23; O 22). Ein Konsens findet sich, trotz zwiespältiger Haltung zur Rolle von Werten im Bankgeschäft (insbesondere beim Thema Rüstung; B 53), beim Thema Verantwortung in Bezug auf die Art der Renditeerzielung:

Damals war dann noch: ,Ja, und außerdem machen wir dann auch gesellschaftlich Verantwortung.“ Und da ist halt die klare Logik der letzten Jahre: „Nein, das Eine kann man nicht vom Anderen trennen. Wir können nicht Rendite in einer verantwortungslosen Weise erwirtschaften, sondern eine Renditeerwirtschaftung muss auch immer mit einer bestimmten Verantwortungskomponente verbunden sein.“ (B 53)

Spezifiziert und erweitert wird die Vorstellung von Verantwortlichkeit unter Rückgriff auf das Bild des ehrbaren Kaufmanns (R 89) oder auf globale Standards nachhaltigen Investierens von Banken (J 31), sodass sich dadurch bestimmte Praktiken, wie zum Beispiel Nahrungsmittelspekulationen oder Rüstungsfinanzierungen ausschließen:

Und außerdem arbeiten wir halt noch mit diesen Ausschlusskriterien, weil wir [...] sagen, wir wollen eben mit bestimmten Geschäftsfeldern von Unternehmen einfach nichts zu tun haben. (G 16)

[...] wir haben ja angefangen zum Beispiel, uns auch aus so was wie Agrarspekulationen und so was als Bank herauszuziehen, [...] im Sinne der Nachhaltigkeit verzichten wir trotzdem auch irgendwo auf Gewinne oder andere Dinge, die wir aus ertragreichen Feldern ziehen können. Oder wir nehmen ja auch in der Kreditvergabe oder Ähnliches längst nicht mehr jeden Kunden. [...] man verzichtet irgendwo ein bisschen auf den ökonomischen Benefit zugunsten der Nachhaltigkeit. (D 29)

Bei diesen Entscheidungen ist die Deutung leitend, dass eine Bank Verantwortung übernehmen kann und soll für die Art und Weise wie und wohin das Geld fließt:

Wir haben ein Haus hier in [...], damit retten wir garantiert nicht die Welt. Und wir retten auch natürlich mit unserem Asset Management nicht die Welt, muss man auch dazusagen. Aber wir tun ... ich glaube, das ist unser größter Beitrag. Und ich glaube, ich muss immer fragen [...]: Wo trage ich die größte Verantwortung für? Und wir geben mehrere Milliarden auf dem Kapitalmarkt zurück, das ist unsere Hauptverantwortung. Und da ist das Thema auf jeden Fall angekommen auch in den Strukturen in der Form. (C 21)

Nachhaltigkeit scheint also den Effekt zu haben, dass sich Banken durch die Verwaltung von Geld, das eben nicht als neutral verstanden wird, ihrer Verantwortung bewusst werden (müssen) und das nutzen, indem sie auf diese Weise einen Kompro- 
miss zwischen Nachhaltigkeit und dem eigenen Geschäftsmodell finden. Dies zeigt sich auch in der Umsetzung von globalen Standards wie den Equator Principles ${ }^{4}$.

[...] Also, ich will jetzt die [Bank J] nicht als nachhaltige Bank bezeichnen, aber als eine Bank, die sich um Nachhaltigkeitsthemen in den Prozessen und Strukturen bemüht, ja, [...] eben, dass man sich solche Leitplanken setzt und sagt: „Wir machen einfach nicht jede Art von Finanzierung." Das ist für mich das wirklich Entscheidende. [...] Und das wäre eben im Finanzierungsbereich und das ist immer das Kerngeschäft, sind das eben die Weltbankstandards oder die Equator Principles. Ja, da kann man darüber kritisieren, auch mit Recht, die Weltbank hat auch nicht alles richtig gemacht bei großen Projektfinanzierungen. Aber wenn ich dahin komme zu sagen ,Ich habe mir für alle meine Tätigkeitsbereiche genau überlegt, an welchen ESG-Standards ich mich orientieren möchte", dann ist eigentlich schon viel ... oder das Optimum erreicht. (J 31)

Der Ansatz ist nicht, ganz bestimmte Werte auszuleben, sondern eine Verantwortung in Bezug auf die Gesellschaft, die man durch die Lenkung von Geldflüssen hat, wahrzunehmen und sich zu positionieren.

\subsubsection{Handlungsregel}

Die durch die Analyse hervortretende Handlungsregel, mit der Akteure auf diese Deutung reagieren können, lautet: Nutze deinen Gestaltungsspielraum und nimm Verantwortung für die Gesellschaft wahr oder zeige, wo und wie du Verantwortlichkeit lebst. Indem die Verantwortung für die Gesellschaft, die über die reine zweckmäßige Erfüllung der Kapitalbereitstellung hinausgeht, angenommen und in einem gewissen Rahmen neu auszugestalten versucht wird, kann das von den Banken definierte Problem des verantwortungslosen Verhaltens vor der Finanzmarktkrise mit entsprechenden Praktiken und Strukturen (bspw. Verkaufs- und Beratungspraxis oder Spekulationen) gelöst werden. Zu dieser Verantwortung zählt auch, sich Gedanken zu machen, wohin das Eigenkapital fließt oder auch, an wen Kredite ausgegeben werden und dazu transparent Position zu beziehen.

Einige Banken nehmen diese Probleme als Anlass, ihre Verantwortung so wahrzunehmen, dass sie die bestehenden Strukturen und Denkweisen überwinden wollen und sich so wieder als legitime Akteure in der Gesellschaft darstellen können. Die Potenzierung dieser Handlungsregel ist folglich eine völlige Umkehr vom gedeuteten Problem: weg vom Verständnis als Verursacher der Krise, die der gesamten Gesellschaft geschadet hat, hin zum Wunsch, eine führende Rolle beim gesellschaftlichen Wandel für eine nachhaltigere Welt einzunehmen.

Weil sie entscheiden, gebe ich dem den Kredit oder dem den Kredit. Und dieser Kredit hat eine Laufzeit von 20-30 Jahren. Das heißt, ich finanziere heute Dinge, die Auswirkungen für die nächsten 20-30 Jahre für unsere Gesellschaft

\footnotetext{
${ }^{4}$ Die Equator Principles sind ein freiwilliges, international etabliertes Rahmenwerk für nachhaltiges Risikomanagement bei Projektfinanzierungen von Geschäftsbanken (siehe dazu Brönner und Lehmann 2016).
} 
haben. Und damit kann ich als Bank ... habe ich eine Schlüsselrolle bei dem Thema Transformation unserer Gesellschaft. [...] Und das müssen sie als Gestaltungsspielraum nutzen. (P 76)

Ein Teil der interviewten Banken (und nicht nur die ausschließlich nachhaltigen Banken) verstehen sich nämlich als Treiber gesellschaftlichen Wandels; sie wollen die Gesellschaft nachhaltig umstellen (I 223) und das Finanzsystem grundlegend verändern (u.a. L 7). Es geht den Banken darum, in der Gesellschaft etwas zu bewirken (P 44) und das Thema Nachhaltigkeit voranzubringen (C 118; D 87); selbst gegen mögliche Widerstände innerhalb der Bank (K 105-108; P 26-41):

Ja, es ist der gesellschaftliche Wandel, den wir jetzt mit aus meiner Sicht gestalten können. Und ich finde, wenn man zur Entfaltung dran ist, jetzt ... jetzt ist eigentlich noch viel Zeit, jetzt können wir ihn mit gestalten. Ich sage ja immer: Schlimmer ist es, getrieben zu sein. Dann trage ich lieber ein bisschen selbst mit auch. Wenn es manchmal schwer ist, wenn es viel Überzeugungskraft erfordert in der Anfangsphase ... (K 108)

Gerade diese Potenzierung der Handlungsregel zeigt die Aktualität und das groBe Legitimitätspotential dieses Deutungsmusters - vom Überwinden der Kritik der Verantwortungslosigkeit hin zum Antreiber gesellschaftlichen Wandels.

\section{3 ,Richtige“ Rendite}

Das dritte aus dem Interviewmaterial rekonstruierte Deutungsmuster problematisiert die reine Renditemaximierung. Banken versuchen aus dieser Perspektive heraus, ihre Legitimationskrise dadurch zu überwinden, dass sie ihr Renditestreben nicht als Selbstzweck, sondern als mit ihren Wertorientierungen im Einklang stehend begreifen. Sie deuten ihre Rendite als ,richtig“, weil sie ökonomisch notwendig und an andere Werte gebunden ist. Die Handlungsregel lautet hier: Löse den Widerspruch zwischen Rendite- und Wertorientierung auf.

\subsubsection{Problemdefinition}

Dieses Deutungsmuster wird durch das Problem bestimmt, dass Banken das ,richtige"Verhältnis zwischen den gegensätzlichen Polen Renditestreben und Wertorientierung finden müssen, da die bisherige Profitmaximierung diskreditiert erscheint. Auf der einen Seite sind sie als Unternehmen dazu angehalten, Rendite zu erwirtschaften; auf der anderen Seite werden reines Renditestreben und Profitmaximierung aber als illegitim und schädlich erachtet (siehe auch Deutungsmuster 1). Sie ringen daher um die richtige, weil legitime Auffassung von Rendite; sie ringen um das „richtige“ Verhältnis von Renditestreben und Wertorientierung. Banken müssen aber durch ein Bekenntnis zum Renditestreben belegen, dass sie keine philanthropischen Einrichtungen sind, sondern Unternehmen, die selbst unter dem Label der Nachhaltigkeit gewinnorientiert arbeiten müssen. Schließlich bildet Rendite die Basis unternehmerischen Handels (u.a. A 8; C 57-66; L 42-45, 48-53; N 78; P 172-184). 
Und also wir sind hier keine gemeinnützige Organisation, sondern wir haben hier einen ökonomischen Auftrag, einen privatwirtschaftlichen Auftrag. Das ist auch, finde ich, sehr gut und sehr in Ordnung. Und damit müssen wir jetzt überlegen, wie wir das Thema Nachhaltigkeit sinnvollerweise ausfüllen. (B 23) Dahinter steht natürlich der Gedanke: „Gewinn ist nicht alles, aber ohne Gewinn ist alles nichts. “ Das heißt also, ein Unternehmen kann keine Arbeitsplätze zur Verfügung stellen, wenn es nicht wirtschaftlich erfolgreich ist. [...] Und ich glaube, das ist auch ein sehr vernünftiger Ansatz, dass man langfristig versucht, positiv sozusagen mehr Erträge als Kosten zu haben. Wenn es umgekehrt wäre, dann wäre das schrecklich. (B 53)

Marktgesetze können auch von Banken mit alternativen Geschäftsmodellen oder bestimmten Wertorientierungen nicht außer Kraft gesetzt werden. Rendite ist schlicht notwendig, um als Bank zu bestehen. Wie wichtig einerseits die Anerkennung als Marktakteur ist und man andererseits kein illegitimes Renditestreben vorgeworfen bekommen möchte, sieht man an folgenden Aussagen:

Für uns war neben natürlich ... ich meine, wir sind eine Bank, wir wollen natürlich auch alle, dass unsere Gehälter bezahlt werden, dass unsere Stromrechnung bezahlt wird, dass wir hier mal in den Urlaub fahren können oder so. Natürlich müssen wir auch Gewinne erzielen, damit die Bank weiter bestehen kann. Großes Ziel ist es aber trotzdem auch immer von der Bank gewesen, dass wir mit unserer Arbeit auch einen hohen sozialen Nutzen auch stiften wollen. (R 20)

Wir sind eine Nachhaltigkeitsbank, wir machen Gewinne, oder wir wollen Gewinne erzielen. Nur so können wir auch nachhaltig im Markt bestehen. Also, es geht nicht um Philanthropie. Es sind marktübliche Zinsen, die wir verlangen, aber auch marktübliche Zinsen, die wir an unsere Kunden in Form von Zinsen auf ihr Tagesgeld oder ähnliches weitergeben. (L 10)

\subsubsection{Wertvorstellung}

Mit dem Deutungsmuster ist die Wertvorstellung verbunden, dass Rendite als Selbstzweck wenig wünschenswert ist; zusätzlich bedarf es einer irgendwie gearteten Wertorientierung (C 57-66; L 48-53; M 4-9; P 172-180; R 20). Es gilt jedoch weiterhin, dass Banken Rendite erwirtschaften müssen und wollen, damit sie als Unternehmen wahrgenommen werden. Daher wird es innerhalb des Deutungsmusters als notwendig erachtet, Rendite - auch unabhängig von Nachhaltigkeit - mit einer Wertorientierung zu verbinden.

Die Kür liegt darin, am Ende beides gut hinzubekommen. (H 34)

Und so einen Antritt zu sagen, es kommt letztlich immer nur darauf an, egal wo irgendwie Geld zu verdienen, ist uns fremd, und darauf haben wir nie das Unternehmen gegründet. (M 7) 
Im Zweifelsfall werden deshalb renditeversprechende Geschäfte nicht durchgeführt, wenn sie den Werten der Bank entgegenstehen (C 57-66; L 11, 48-53). Eine reine Renditeorientierung wird dementsprechend negativ bewertet (u. a. K 95):

Und eben auch die Frage, lebe ich ein gewisses Werteverständnis in der Bank als Nachhaltigkeit oder bin ich wie in einer konventionellen Bank rein renditegetrieben. (E 21)

Konventionelle Banken [...] haben eben immer diesen monetären Ansatz, wie die Gewinnmaximierung stattfinden kann. Und bei diesen Alternativbanken [...] stehen halt auch andere Ziele noch im Vordergrund. (G 21)

\subsubsection{Handlungsregel}

Als Reaktion auf diese Deutung wird durch die Analyse des Materials folgende Handlungsregel offensichtlich: Löse den Widerspruch zwischen Rendite- und Wertorientierung auf. Mit dieser Orientierung können Banken die notwendige Rendite erzielen, ohne sie ausschließlich als Selbstzweck zu verstehen. Auf diese Weise können sich Kreditinstitute als legitime Akteure darstellen, denn sie sind als Unternehmen erfolgreich, ohne auf das Verfolgen gesellschaftlicher Werte zu verzichten.

Nachhaltigkeit wird daher innerhalb dieses Deutungsmusters logisch konsistent so gedeutet, dass damit kein Renditeverzicht einhergeht (O 21, 77-84) und auf lange Sicht eine Rendite erreicht wird, ,die sich auch blicken lassen kann“ (O 35). Zwischen Wertorientierung und Renditestreben besteht kein Widerspruch, der aufgelöst werden müsste. Mit Blick auf das vorgelegte Diagramm, in dem die Interviewten verschiedene Banken unter anderem auf der Achse von werte- bis renditeorientiert verorten sollten, zeigt sich diese Deutung exemplarisch:

Die [Grafik] hat für mich nämlich tatsächlich einen Widerspruch, weil, wir glauben nicht, dass Wertorientierung und Renditeorientierung sich extrem gegenüberstehen. Oder gegeneinander agieren. Dass man halt sagt, wenn man wertebasiert vorgeht, definitiv eine schlechtere Rendite hat, insbesondere erst mal rein bei der Kapitalanlage. [...] Von daher sind wir da voll und ganz davon überzeugt, dass es halt auch etwas Gutes ist, und so gehen wir auch an unsere Kunden ran. (O 21)

Auch in einem Verständnis von Nachhaltigkeit als einem business case im Bankwesen zeigt sich die Möglichkeit, Rendite in Verbindung mit einer Wertorientierung zu erlangen. Indem nachhaltiges Handeln die Reputationsrisiken (u.a. N 87) und die Risikoausfallkosten (C 14, 57-66; G 43) senkt und somit zu einem besseren Risikomanagement beiträgt (B 26), lässt es sich auch in das Geschäftsmodell konventioneller Banken integrieren, weil es eben das Gesicht einer positiven Werthaltung mit sich bringt. Zudem kann Nachhaltigkeit für weitere Produkte und Wettbewerbsvorteile auf Seiten der Bank sorgen (B 26; C 10-11; J 24; K 93; Q 51). Rendite steht Nachhaltigkeit demnach nicht entgegen, sondern kann sogar als Folge davon betrachtet werden: 
Und wir glauben, dass Rendite nicht das Ziel ist, sondern das Ergebnis von nachhaltigem Handeln. Das ist die logische Konsequenz daraus. Das heißt, die Unternehmen, mit denen wir zusammenarbeiten, schaffen es durchaus, ein nachhaltiges Geschäftsmodell zu haben, auch wenn sie vielleicht in Bereichen tätig sind, wo viele Leute glauben, da kann man kein Geld mit verdienen. Das kann man sehr wohl [...]. (L 11)

Entgegen der Auffassung, dass Rendite die Konsequenz aus nachhaltigem Bankwesen darstellt (L 11), wird auch betont, dass Nachhaltigkeit zunächst Kosten verursacht (Q 47-51). Durch den Ausschluss bestimmter Geschäftsfelder verzichtet man zudem auf die dort möglichen Gewinne (D 30). Aufgrund der legitimitätsstiftenden Wirkung, die Rendite zugesprochen wird, reicht es daher nicht aus, auf diese Deutung von Rendite allein durch Verweis auf eine Wertorientierung zu reagieren. Mit Blick auf die mit einem Renditeverzicht verbundenen Befürchtungen wird deutlich, dass es einer Auflösung des Widerspruchs zwischen Rendite- und Wertorientierung bedarf. Sie sind letztlich Ausweis der legitimierenden Wirkung, die Rendite für Banken mit sich bringt:

[...] die wollen vor allem eine Rendite haben, das muss man ja auch ganz klar sagen und müssen die Altersvorsorge für ihre Priester sichern oder müssen den laufenden Betrieb am Leben erhalten. Und da gibt es immer wieder diese Zweifel: „Ist das nicht ein Performance-Verzicht, letztendlich ein Rendite-Verzicht?“ (P 35)

\section{Diskussion}

Wie sollte eine Bank sein, was ist ihre Aufgabe, für wen sollte sie handeln? Mit solchen Fragen müssen sich Kreditinstitute spätestens seit Beginn der Finanzkrise vermehrt auseinandersetzen. Nachdem sich bestimmte Geschäftspraktiken und Strukturen als problematisch, illegitim und illegal erwiesen haben, sind Banken auf der Suche nach Geschäftsmodellen, die einträglich und legitimitätsstiftend zugleich sind. Sie versuchen sich als gesellschaftliche Akteure (Hiß und Nagel 2017) in stetigen Aushandlungsprozessen mit verschiedenen Teilen der Gesellschaft als Unternehmen zu verorten, die einen positiven Beitrag zum Zusammenleben leisten (siehe dazu die Beiträge in Wendt 2016). Da in solchen Umbruchs- und Krisensituationen, wie sie Banken derzeit erfahren, durch die Entstehung des Neuen eine Auseinandersetzung mit dem Alten einhergeht, sind diese Umstände ideal für die Rekonstruktion von Deutungsmustern (Meuser und Sackmann 1992a, S. 20).

Sichtbar wird die Auseinandersetzung zwischen Altem und Neuem besonders daran, welche Rolle dem Thema Nachhaltigkeit in den Deutungen zukommt und wie Banken ihre Rolle in der Gesellschaft verstehen. Nachhaltigkeit erweist sich dort als Gegenpol zu einer maßlosen Renditeorientierung, die den „schlechten“ Banken zugeschrieben und die für die Finanzkrise verantwortlich gemacht wird. Die große gesellschaftliche Akzeptanz von Nachhaltigkeit und der damit verbundene moralische Anspruch bieten scheinbar eine einfache, aber effektive Möglichkeit, sich von 
dem Krisenhaften der Bankenwelt abzugrenzen. Überhaupt zeigen die Problemdefinitionen, dass Banken mit moralischen Fragen konfrontiert werden, sodass sie nicht nur als rationale Akteure auftreten können. Es erscheint weniger bedeutsam, was Nachhaltigkeit konkret bedeutet oder wie es letztlich umgesetzt wird, da allein die Abkehr von reinem Renditestreben die notwendige Legitimität für Banken zu versprechen scheint. Es zeigt sich auch die Widersprüchlichkeit, die Rendite innewohnt. Sie trägt zur Legitimation von Banken bei, kann aber auch zum Bestandteil eines illegitimen Geschäftsmodells werden.

Die Auseinandersetzung zwischen Altem und Neuem zeigt sich auch an der Rolle, die Banken für sich in Anspruch nehmen. Um nicht mit der Krise und den damit verbundenen Problemen und dem ,Schlechten“ an sich in Verbindung gebracht zu werden, stehen ihnen bestimmte Deutungen zur Verfügung: Banken können sich auf der einen Seite als Transformateure der Gesellschaft positionieren, die die Zukunft gestalten wollen und sich auf der anderen Seite auf ihre Wurzeln und die eigentlichen Aufgaben des Bankwesens zurückbesinnen. „Gutes“ Bankwesen orientiert sich demnach daran, wofür es aus Sicht der Banken eigentlich einmal entstanden ist und was es Positives in der Gesellschaft bewirken kann.

Mit Blick auf die Vielfalt des deutschen Bankwesens könnten die vorherrschenden Deutungsangebote zwei gegensätzliche Entwicklungen hervorrufen: Indem Banken sich auf ihren Ursprung und ihre Wurzeln zurückbesinnen, und sich auf diese Weise von der gegenwärtigen Krise absetzen, könnte die Differenzierung zwischen den Banktypen wieder deutlicher zutage treten. Inwiefern diesen Deutungen entsprechende Handlungen folgen, müsste jedoch auf eine andere Weise ermittelt werden.

\section{Fazit}

Mit unserer Analyse haben wir untersucht, wie sich Banken als Reaktion auf die Krise legitimieren. Die Rekonstruktion von Deutungsmustern auf Basis von 18 diskursiven Interviews mit deutschen Banken hat Deutungen hervorgebracht, die zu einem besseren Verständnis vom Umgang der Kreditinstitute mit den vielfältigen Krisen und der zur Disposition stehenden Legitimation beitragen. Da wir die Deutungen aller Banktypen erhoben haben, geben die Ergebnisse einen Einblick in die gegenwärtigen Bewusstseinsstrukturen deutscher Banken bei ihrem Versuch, die Krisen hinter sich zu lassen. Mit der Abgrenzung von anderen Banken, dem Fokus auf die eigene Verantwortung und dem Präsentieren der ,richtigen“ Rendite zeigen die Deutungsmuster der Banken, welche Probleme überhaupt wahrgenommen werden, welche Wertvorstellungen bestehen und welche Handlungsregeln zur Lösung der Probleme beitragen sollen.

Die Deutungsmuster zeigen, dass im Kontext von Nachhaltigkeit neue Strategien gefunden werden, um sich abzugrenzen und moralisch überlegen darzustellen. An diesem Deutungsangebot, dass sich im Kontext von Nachhaltigkeit zeigt, können sich letztlich alle Banken bedienen und entsprechend $\mathrm{zu}$ legitimieren versuchen. Nachhaltigkeit ist nicht immer Ziel an und für sich, sondern wird häufig nur als Mittel zum Zweck betrachtet. Banken, die im Zuge der Krise diskreditiert wurden, können sich durch den Bezug zur Nachhaltigkeit auf ein Bankwesen besinnen, das 
nicht den Geruch der Krise trägt. Ob Handlungen, die auf einem solchen Deutungsangebot basieren, letztlich künftige Krisen verhindern können, indem sie Banken und deren Geschäftsmodelle krisenfester machen, bleibt jedoch fraglich. Ob solche Handlungen überhaupt in großem Maße Einzug ins Bankwesen halten, bleibt zudem abzuwarten.

Danksagung Der vorliegende Beitrag ist entstanden im Forschungsprojekt „Doppelte Dividende. Beitrag des nachhaltigen Investierens zur Stabilisierung des Finanzmarkts“, das vom Bundesministerium für Bildung und Forschung (BMBF) im Rahmen der Förderinitiative „Finanzsystem und Gesellschaft“ von 2015 bis 2018 gefördert wurde. Die vorliegenden Forschungsergebnisse wurden präsentiert und diskutiert auf dem 33. Colloquium der European Group for Organization Studies in Kopenhagen 2017, dem 112. Annual Meeting der American Sociological Association in Montreal 2017 sowie auf dem Workshop „Privatanleger auf dem Finanzmarkt“ in Frankfurt am Main 2018. Wir danken dem BMBF, allen Diskutierenden für ihre konstruktiven Hinweise, den anonymen Gutachtern und allen Interviewpartnern.

Open Access Dieser Artikel wird unter der Creative Commons Namensnennung 4.0 International Lizenz (http://creativecommons.org/licenses/by/4.0/deed.de) veröffentlicht, welche die Nutzung, Vervielfältigung, Bearbeitung, Verbreitung und Wiedergabe in jeglichem Medium und Format erlaubt, sofern Sie den/die ursprünglichen Autor(en) und die Quelle ordnungsgemäß nennen, einen Link zur Creative Commons Lizenz beifügen und angeben, ob Änderungen vorgenommen wurden.

\section{Literatur}

Beyer, Jürgen, und Markus Wolf. 2014. Finanzialisierung in der Finanzmarkt-Kommunikation deutscher Großbanken. In Politische Ökonomie der Finanzialisierung, Hrsg. Marcel Heires und Andreas Nölke, 131-146. Wiesbaden: Springer VS.

Bögelein, Nicole. 2016. Deutungsmuster von Strafe. Eine strafsoziologische Untersuchung am Beispiel der Geldstrafe. Wiesbaden: Springer VS.

Brönner, Achim, und Sabine Lehmann. 2016. Equator Principles - Risikomanagement bei Finanzierungen Die EP - ihr Anspruch, ihre Verbreitung, ihre Wirkung. In CSR und Investment Banking. Investment und Banking zwischen Krise und Positive Impact, Hrsg. Karen Wendt, 93-113. Berlin: Springer Gabler.

Deephouse, David L., und Mark Suchman. 2008. Legitimacy in organizational institutionalism. In The SAGE handbook of organizational institutionalism, Hrsg. Royston Greenwood, Christine Oliver, Roy Suddaby und Kerstin Sahlin, 49-77. London: Sage.

DiMaggio, Paul J., und Walter W. Powell. 1983. The iron cage revisited. Institutional isomorphism and collective rationality in organizational fields. American Sociological Review 48:147-160.

Hauff, Volker. Hrsg. 1987. Unsere gemeinsame Zukunft. Der Brundtland-Bericht der Weltkommission für Umwelt und Entwicklung. Greven: Eggenkamp.

Herzog, Lisa, Sarah Lenz und Edgar Hirschmann. 2015. Ethische Banken in Deutschland - Nische oder Avantgarde? Eine Analyse der Selbstdarstellungen alternativer Geldinstitute. IfS Working Paper. Frankfurt a. M. Institut für Sozialforschung.

Hiß, Stefanie. 2006. Warum übernehmen Unternehmen gesellschaftliche Verantwortung? Ein soziologischer Erklärungsversuch. Frankfurt a. M.: Campus.

Hiß, Stefanie. 2011. Globale Finanzmärkte und nachhaltiges Investieren. In Handbuch Umweltsoziologie, Hrsg. Matthias Groß, 651-670. Wiesbaden: VS Verlag für Sozialwissenschaften.

Hiß, Stefanie. 2012. Konfligierende Rationalitäten - wie Nachhaltigkeit die Rationalitätsordnung des Finanzmarktes irritiert. In Wirtschaftliche Rationalität. Soziologische Perspektiven, Hrsg. Anita Engels und Lisa Knoll, 85-107. Wiesbaden: Springer VS.

Hiß, Stefanie, und Sebastian Nagel. 2017. Unternehmen als gesellschaftliche Akteure. In Handbuch der Wirtschaftssoziologie, Hrsg. Andrea Maurer, 331-348. Wiesbaden: Springer VS.

Hiß, Stefanie, Sebastian Nagel und Bernd Teufel. 2018. Gesellschaftliche Verantwortung von Unternehmen und ihren Investoren: Zur Geschichte einer gemeinsamen Infrastruktur. In Gesellschaftliche Verantwortung von Unternehmen in Deutschland: Ein Kompendium zur Erschließung eines sich entwickelnden Themenfeldes, Hrsg. Holger Backhaus-Maul, Martin Kunze und Stefan Nährlich, 67-86. Wiesbaden: Springer VS. 
Honegger, Claudia, Sighard Neckel und Chantal Magnin. 2010. Strukturierte Verantwortungslosigkeit. Berichte aus der Bankenwelt. Berlin: Suhrkamp.

Jepperson, Ronald L. 1991. Institutions, institutional effects, and institutionalism. In The new institutionalism in organizational analysis, Hrsg. Walter W. Powell und Paul J. DiMaggio, 143-163. Chicago, IL: University of Chicago Press.

Lüders, Christian. 1991. Deutungsmusteranalyse. Annäherungen an ein risikoreiches Konzept. In Qualitativ-empirische Sozialforschung. Konzepte, Methoden, Analysen, Hrsg. Detlef Garz und Klaus Kraimer, 377-408. Opladen: Westdeutscher Verlag.

Lüders, Christian, und Michael Meuser. 1997. Deutungsmusteranalyse. In Sozialwissenschaftliche Hermeneutik. Eine Einführung, Hrsg. Ronald Hitzler und Anne Honer, 57-79. Opladen: Leske + Budrich.

Lütz, Susanne. 2017. Finanzmärkte. In Handbuch der Wirtschaftssoziologie, Hrsg. Andrea Maurer, 385-413. Wiesbaden: Springer VS.

Markova, Hristina. 2013. Exzellenz durch Wettbewerb und Autonomie? Deutungsmuster hochschulpolitischer Eliten am Beispiel der Exzellenzinitiative. Konstanz: UVK.

Meuser, Michael. 2011. Deutungsmusteranalyse. In Hauptbegriffe Qualitativer Sozialforschung, Hrsg. Ralf Bohnsack, Winfried Marotzki und Michael Meuser, 31-33. Opladen: Barbara Budrich.

Meuser, Michael, und Reinhold Sackmann. 1992a. Zur Einführung. Deutungsmusteransatz und empirische Wissenssoziologie. In Analyse sozialer Deutungsmuster. Beiträge zur empirischen Wissenssoziologie, Hrsg. Michael Meuser und Reinhold Sackmann, 9-37. Pfaffenweiler: Centaurus-Verlagsgesellschaft.

Meuser, Michael, und Reinhold Sackmann. Hrsg. 1992b. Analyse sozialer Deutungsmuster. Beiträge zur empirischen Wissenssoziologie. Pfaffenweiler: Centaurus-Verlagsgesellschaft.

Meyer, John W., und Brian Rowan. 1977. Institutionalized organizations. Formal structure as myth and ceremony. American Journal of Sociology 83:340-363.

Münnich, Sascha. 2016. Readjusting imagined markets. Morality and institutional resilience in the German and British bank bailout of 2008. Socio-Economic Review 14:283-307.

Oevermann, Ulrich. 2001a. Zur Analyse der Struktur von sozialen Deutungsmustern. Sozialer Sinn 2:3-33.

Oevermann, Ulrich. 2001b. Die Struktur sozialer Deutungsmuster - Versuch einer Aktualisierung. Sozialer Sinn 2:35-82.

Opielka, Michael, Matthias Müller, Tim Bendixen und Jesco Kreft. 2010. Grundeinkommen und Werteorientierungen. Eine empirische Analyse. 2., korrig. Aufl. Wiesbaden: VS Verlag für Sozialwissenschaften.

Rethel, Lena, und Timothy J. Sinclair. 2012. The problem with banks. London: Zed Books.

Rona-Tas, Akos, und Stefanie Hiß. 2010. The role of ratings in the subprime mortgage crisis. The art of corporate and the science of consumer credit rating. In Research in the sociology of organizations. Special issue on markets on trial: The economic sociology of the U.S. financial crisis, Hrsg. Michael Lounsbury und Paul M. Hirsch, 115-155. Bingley: Emerald.

Sächsische Carlowitz-Gesellschaft. Hrsg. 2013. Die Erfindung der Nachhaligkeit. Leben, Werk und Wirkung des Hans Carl von Carlowitz. München: oekom Verlag.

Sachverständigenrat zur Begutachtung der Gesamtwirtschaftlichen Entwicklung. Hrsg. 2008. Das deutsche Finanzsystem Effizienz steigern - Stabilität erhöhen. Expertise im Auftrag der Bundesregierung. Wiesbaden: Statistisches Bundesamt.

Sachweh, Patrick. 2010. Deutungsmuster sozialer Ungleichheit. Wahrnehmung und Legitimation gesellschaftlicher Privilegierung und Benachteiligung. Frankfurt a. M.: Campus.

Schütze, Yvonne. 1992. Das Deutungsmuster „Mutterliebe“ im historischen Wandel. In Analyse sozialer Deutungsmuster. Beiträge zur empirischen Wissenssoziologie, Hrsg. Michael Meuser und Reinhold Sackmann, 39-48. Pfaffenweiler: Centaurus-Verlagsgesellschaft.

Scott, William Richard. 2008. Institutions and organizations. Ideas and interests. Los Angeles, CA: Sage.

Senge, Konstanze. 2006. Zum Begriff der Institution im Neo-Institutionalismus. In Einführung in den NeoInstitutionalismus, Hrsg. Konstanze Senge und Kai-Uwe Hellmann, 35-47. Wiesbaden: VS Verlag für Sozialwissenschaften.

Senge, Konstanze. 2011. Das Neue am Neo-Institutionalismus. Der Neo-Institutionalismus im Kontext der Organisationswissenschaft. Wiesbaden: VS Verlag für Sozialwissenschaften.

Soule, Sarah A. 1997. The student divestment movement in the United States and tactical diffusion. The shantytown protest. Social Forces 75:855-883.

Stiehl, Martina. 1989. Die Ökobank-Bewegung. Zeitschrift für das gesamte Genossenschaftswesen 39:24-30.

Suchman, Mark C. 1995. Managing legitimacy. Strategic and institutional approaches. Academy of Management Review 20:571-610. 
Toppinen, Anne, Aino Virtanen, Audrey Mayer und Anni Tuppura. 2015. Standardizing social responsibility via ISO 26000. Empirical insights from the forest industry. Sustainable Development 23:153-166.

Ullrich, Carsten G. 1999. Deutungsmusteranalyse und diskursives Interview. Zeitschrift für Soziologie 28:429-447.

Walgenbach, Peter, und Renate E. Meyer. 2008. Neoinstitutionalistische Organisationstheorie. Stuttgart: Kohlhammer.

Weber, Beat, und Stefan W. Schmitz. 2011. Varieties of helping capitalism. Politico-economic determinants of bank rescue packages in the EU during the recent crisis. Socio-Economic Review 9:639-669.

Wendt, Karen. Hrsg. 2016. CSR und Investment Banking. Investment und Banking zwischen Krise und Positive Impact. Berlin: Springer Gabler.

Windolf, Paul. 2005. Was ist Finanzmarkt-Kapitalismus? In Finanzmarkt-Kapitalismus. Analysen zum Wandel von Produktionsregimen, Hrsg. Paul Windolf, 20-57. Wiesbaden: VS Verlag für Sozialwissenschaften.

Woll, Cornelia. 2014. The power of inaction. Bank bailouts in comparison. Ithaca, NY: Cornell University Press.

Stefanie Hiß 1974, Prof. Dr. rer. pol., Lehrstuhlinhaberin für Soziologie mit dem Schwerpunkt Märkte, Organisationen und Governance, Friedrich-Schiller-Universität Jena. Forschungsgebiete: Nachhaltigkeit, Corporate Social Responsibility, Finanzmarkt, Finanzialisierung, Rating, Institutionentheorien, Frauen in Führungspositionen, soziale Ungleichheit, Stadtsoziologie. Veröffentlichungen: Warum übernehmen Unternehmen gesellschaftliche Verantwortung. Ein soziologischer Erklärungsversuch. Frankfurt a. M. 2006; The Politics of the Financialization of Sustainability. In: Competition \& Change 17 (3): S. 234-247, 2013.

Gesa Griese 1989, M.A., wissenschaftliche Mitarbeiterin, Lehrstuhl für Soziologie mit dem Schwerpunkt Märkte, Organisationen und Governance, Friedrich-Schiller-Universität Jena, Forschungsgebiete: Organisations- und Wirtschaftssoziologie, Finanzmarkt und Nachhaltigkeit, Nachhaltiges Bankwesen, Entrepreneure von Nachhaltigkeit im deutschen Bankwesen.

Sebastian Nagel 1986, M.A., wissenschaftlicher Mitarbeiter, Lehrstuhl für Soziologie mit dem Schwerpunkt Märkte, Organisationen und Governance, Friedrich-Schiller-Universität Jena, Forschungsgebiete: Wirtschaftssoziologie, Organisations- und Unternehmenssoziologie, Soziologie der Finanzmärkte, Nachhaltige Finanzmärkte, Soziologischer Neo-Institutionalismus. Veröffentlichungen: Ratingagenturen zwischen Krise und Regulierung. Baden-Baden 2012 (mit S. Hiß); Unternehmen als gesellschaftliche Akteure. In: Handbuch der Wirtschaftssoziologie. Wiesbaden 2017 (mit S. Hiß; hrsg. von A. Maurer). 